\title{
Three new physalins from Physalis alkekengi var. franchetii
}

\author{
Wan-Xuan Xu, ${ }^{\text {a,b }}$ Jian-Chao CHEN, ${ }^{a}$ Jie-Qing LiU, ${ }^{a}$ Lin ZHou, ${ }^{a}$ Yi-Fen WANG, ${ }^{a}$ and Ming-Hua QIU ${ }^{\mathrm{a}, \mathrm{b}, *}$

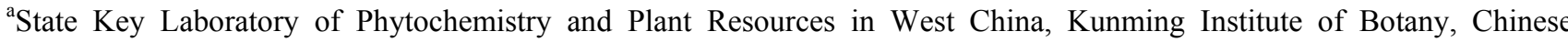 \\ Academy of Sciences, Kunming 650201, China \\ ${ }^{\mathrm{b}}$ University of Chinese Academy of Sciences, Beijing 100049, China
}

Received 10 March 2013; Accepted 3 May 2013

(C) The Author(s) 2013. This article is published with open access at Springerlink.com

\begin{abstract}
Three new physalin steroids, physalin III (1), physalin IV (2), 3-O-methylphysalin X (3), together with five known physalins (4-8) were isolated from the $80 \%$ EtOH extract of calyces of Physalis alkekengi var. franchetii. The structures of the new compounds were revealed through $1 \mathrm{D}$ and 2D NMR and mass spectroscopic studies.
\end{abstract}

Keywords: Physalis alkekengi var. franchetii, steroid, physalin, neophysalin

\section{Introduction}

The genus of Physalis (Solanaceae), including about 120 species, is widely distributed throughout South and North America, which also includes five species, and two variations which also can be found in China ${ }^{1}$. The plant Physalis alkekengi var. franchetii, is used in traditional Chinese medicine ${ }^{2}$, and is chiefly used for the treatment and prevention of tumors, leishmaniasis, sore throat, cough, eczema, hepatitis and urinary problems ${ }^{3}$. The chemical constituents of $P$. alkekengi var. franchetii are mainly composed of alkaloids, flavonoids, sterols, fatty acids, amino acids, and steroids ${ }^{4}$.

Physalins, as one of the characteristic constituents from $P$. alkekengi var. franchetii, is a type of steroids with 16,24cyclo-13,14-seco-ergostane skeleton which has been established by X-ray crystallographic analysis ${ }^{5-7}$. Neophysalins, are another characteristic structure, and are transformed from physalins through an acid-induced benzilic acid-type rearrangement reaction ${ }^{8}$. Until recently, more than 50 physalins and neophysalins have been identified from $P$. alkekegi var. franchetii, $P$. agulata, $P$. lancifolia and $P$. minima. Physalins and neophysalins have tremendous biological activities, including anti-tumor ${ }^{9}$, anti-microbial ${ }^{10}$, anti-malarial $^{11}$, immunosuppressive ${ }^{12}$, anti-inflammatory ${ }^{13,14}$, immunomodulatory $^{15}$, cytotoxic $^{16}$, and trypanocidal ${ }^{17,18}$ effects.

In this study, five physalins $(\mathbf{1}, \mathbf{2}, \mathbf{4}-\mathbf{6})$ and three neophysalins $(\mathbf{3}, \mathbf{7}, \mathbf{8})$ were isolated from $P$. alkekengi var. franchetii, three of which (1-3) were new. Their chemical structures and their elucidation are described herein.

\section{Results and Discussion}

Compound $\mathbf{1}$ was obtained as a white powder. The molecular

*To whom correspondence should be addressed. E-mail: mhchiu@mail.kib.ac.cn

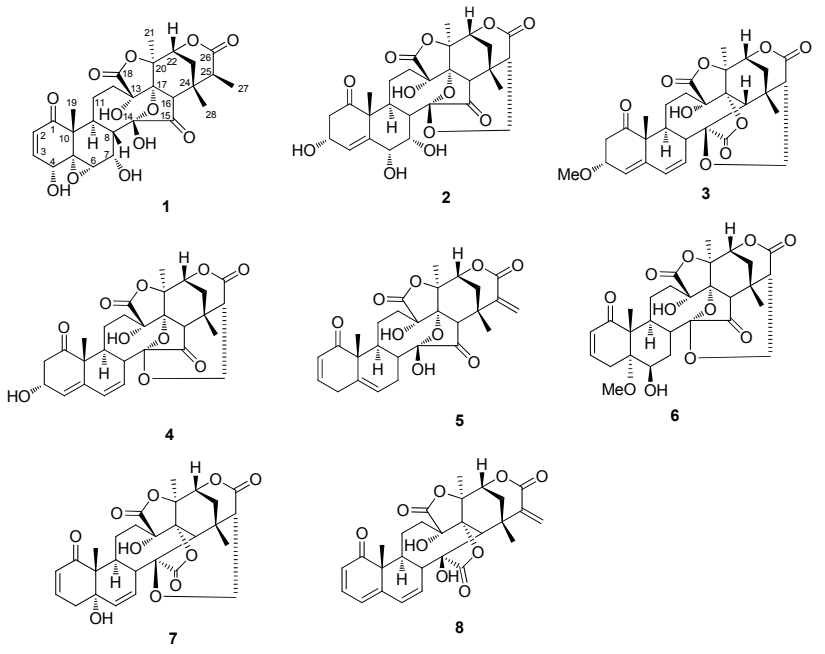

formula of 1 was determined to be $\mathrm{C}_{28} \mathrm{H}_{32} \mathrm{O}_{12}$ by HREIMS $(\mathrm{m} / \mathrm{z} \text { 560.1885, [M] }]^{+}$; calcd 560.1894) and NMR data (Table 1). The ${ }^{1} \mathrm{H}$ NMR spectrum of $\mathbf{1}$ (in pyridine- $d_{5}$ ) showed four methyl signals at $\delta_{\mathrm{H}} 1.29,1.47,1.65$, and 2.02 , four oxygenated signals at $\delta_{\mathrm{H}} 4.01(\mathrm{H}-4), 3.54(\mathrm{H}-6), 5.70(\mathrm{H}-7)$ and 4.73 $(\mathrm{H}-22)$, and two olefinic proton signals at $\delta_{\mathrm{H}} 6.24(\mathrm{H}-2)$ and $7.19(\mathrm{H}-3)$. The ${ }^{13} \mathrm{C}$ DEPT spectrum of 1 (in pyridine- $d_{5}$ ) displayed the presence of twenty-eight carbons, including four methyls, three methylenes, ten methines (two olefinic carbons and four oxymethanes), eleven quaternary carbons (two ketone groups, two lactone groups, four oxygenated carbons, and one ketal carbon). These signals indicated the structural similarity of $\mathbf{1}$ and physalin $\mathrm{J}^{19}$. The chemical shift of the ketal carbon at C-14 $\left(\delta_{\mathrm{C}} 103.4\right)$ of 1 differently from that $\left(\delta_{\mathrm{C}} 107.8\right)$ of physalin $\mathrm{J}$, together with one more methyl signal and less an oxygenated $\mathrm{CH}_{2}$ in 1 indicated that the $\mathrm{C}(27)-\mathrm{O}-\mathrm{C}(14)$ ether 
Table 1. ${ }^{1} \mathrm{H}$ NMR (600 MHz) and ${ }^{13} \mathrm{C}$ DEPT (150 MHz) data of compounds 1-3

\begin{tabular}{|c|c|c|c|c|c|c|}
\hline \multirow[b]{2}{*}{ No. } & \multicolumn{2}{|c|}{ 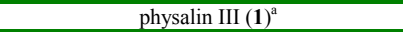 } & \multicolumn{2}{|l|}{ physalin IV (2) ${ }^{\mathrm{a}}$} & \multicolumn{2}{|c|}{ 3-O-methylphysalin X (3) } \\
\hline & $\delta_{\mathrm{H}}(J$ in $\mathrm{Hz})$ & $\delta_{\mathrm{C}}$, type & $\delta_{\mathrm{H}}(J$ in $\mathrm{Hz})$ & $\delta_{\mathrm{C}}$, type & $\delta_{\mathrm{H}}(J$ in $\mathrm{Hz})$ & $\delta_{\mathrm{C}}$, type \\
\hline 1 & & $202.9, \mathrm{C}$ & & $212.3, \mathrm{C}$ & & $213.8, \mathrm{C}$ \\
\hline 2 & $6.24, \mathrm{~d}(9.8)$ & $132.8, \mathrm{CH}$ & $3.12, \mathrm{dd}(11.7,5.4) ; 2.85, \mathrm{dd}(11.7,5.7)$ & $47.1, \mathrm{CH}_{2}$ & $3.13, \mathrm{dd}(13.4,6.5) ; 2.62, \mathrm{~m}$ & $41.2, \mathrm{CH}_{2}$ \\
\hline 3 & $7.19, \mathrm{dd}(9.8,6.5)$ & 145.6, $\mathrm{CH}$ & $4.67, \mathrm{dd}(5.7,5.4)$ & $67.7, \mathrm{CH}$ & $4.42-4.38, \mathrm{~m}$ & $76.7, \mathrm{CH}$ \\
\hline 4 & $4.01, \mathrm{~d}(6.3)$ & $70.3, \mathrm{CH}$ & $6.32, \mathrm{~d}(4.4)$ & $130.5, \mathrm{CH}$ & $5.70, \mathrm{~d}(4.1)$ & $122.4, \mathrm{CH}$ \\
\hline 5 & & $66.2, \mathrm{C}$ & & $145.8, \mathrm{C}$ & & 142. $8, \mathrm{C}$ \\
\hline 6 & 3.54 , br. $\mathrm{s}$ & $62.9, \mathrm{CH}$ & $4.79, \mathrm{~d}(3.8)$ & $77.4, \mathrm{CH}$ & $6.28, \mathrm{dd}(10.4,1.5)$ & $127.5, \mathrm{CH}$ \\
\hline 7 & 5.70, br. s & $65.4, \mathrm{CH}$ & $5.23, \mathrm{dd}(3.5,1.6)$ & $70.6, \mathrm{CH}$ & 6.17, dd $(10.4,3.4)$ & $127.9, \mathrm{CH}$ \\
\hline 8 & $2.93, \mathrm{~m}$ & $44.1, \mathrm{CH}$ & $3.39, \mathrm{dd}(11.7,1.6)$ & $43.8, \mathrm{CH}$ & $2.81, \mathrm{~m}$ & $47.7, \mathrm{CH}$ \\
\hline 9 & $3.45, \mathrm{~m}$ & $32.2, \mathrm{CH}$ & 2.11, dd $(11.7,2.7)$ & $32.2, \mathrm{CH}$ & $2.71-2.74, \mathrm{~m}$ & $32.8, \mathrm{CH}$ \\
\hline 10 & & $49.8, \mathrm{C}$ & & $53.3, \mathrm{C}$ & & $51.0, \mathrm{C}$ \\
\hline 11 & $2.89, \mathrm{~m}$ & $20.4, \mathrm{CH}_{2}$ & $1.63-1.54, \mathrm{~m} ; 2.34, \mathrm{~m}$ & $23.9, \mathrm{CH}_{2}$ & $2.05, \mathrm{~d}(4.4) ; 1.71-1.59, \mathrm{~m}$ & $28.0, \mathrm{CH}_{2}$ \\
\hline 12 & $2.47, \mathrm{~m} ; 2.25-2.31, \mathrm{~m}$ & $32.1, \mathrm{CH}_{2}$ & $2.58, \operatorname{ddd}(16.5,13.0,6.2) ; 1.95, \mathrm{dd}(16.5,9.4)$ & $26.8, \mathrm{CH}_{2}$ & $1.92, \mathrm{~d}(1.3) ; 2.04, \mathrm{~m}$ & $31.9, \mathrm{CH}_{2}$ \\
\hline 13 & & $80.8, \mathrm{C}$ & & $80.3, \mathrm{C}$ & & $80.8, \mathrm{C}$ \\
\hline 14 & & 103.4, C & & 108.5, C & & $82.3, \mathrm{C}$ \\
\hline 15 & & 217.0, C & & 209.7, C & & 169.5, C \\
\hline 16 & $3.23, \mathrm{~s}$ & $55.6, \mathrm{CH}$ & $3.26, \mathrm{~s}$ & $55.3, \mathrm{CH}$ & $2.60, \mathrm{~s}$ & $51.1, \mathrm{CH}$ \\
\hline 17 & & 83.6, C & & $81.9, \mathrm{C}$ & & $83.9, \mathrm{C}$ \\
\hline 18 & & 173.5, C & & 173.7, C & & 173.3, C \\
\hline 19 & $1.65, \mathrm{~s}$ & $15.3, \mathrm{CH}_{3}$ & $1.78, \mathrm{~s}$ & $19.8, \mathrm{CH}_{3}$ & $1.36, \mathrm{~s}$ & $21.2, \mathrm{CH}_{3}$ \\
\hline 20 & & $83.9, \mathrm{C}$ & & $82.6, \mathrm{C}$ & & $82.5, \mathrm{C}$ \\
\hline 21 & $2.02, \mathrm{~s}$ & $22.3, \mathrm{CH}_{3}$ & $2.32, \mathrm{~s}$ & $22.9, \mathrm{CH}_{3}$ & $1.76, \mathrm{~s}$ & $22.3, \mathrm{CH}_{3}$ \\
\hline 22 & 4.73 , br. $\mathrm{s}$ & $77.6, \mathrm{CH}$ & $4.75, \mathrm{~m}$ & $78.0, \mathrm{CH}$ & $4.58, \mathrm{dd}(4.4,1.4)$ & $76.1, \mathrm{CH}$ \\
\hline 23 & $2.14-2.19, \mathrm{~m} ; 1.92, \mathrm{~d}(14.9)$ & $27.5, \mathrm{CH}_{2}$ & $2.11, \mathrm{~m}$ & $33.1, \mathrm{CH}_{2}$ & $1.97, \mathrm{~m}$ & $33.0, \mathrm{CH}_{2}$ \\
\hline 24 & & $36.0, \mathrm{C}$ & & $32.0, \mathrm{C}$ & & $29.7, \mathrm{C}$ \\
\hline 25 & $3.42, \mathrm{~m}$ & $42.4, \mathrm{CH}$ & $3.05, \mathrm{~d}(3.8)$ & $51.2, \mathrm{CH}$ & 2.57, dd $(11.4,2.3)$ & $41.7, \mathrm{CH}$ \\
\hline 26 & & 173.0, C & & 168.1, C & & $169.4, \mathrm{C}$ \\
\hline 27 & $1.29, \mathrm{~d}(7.5)$ & $17.5, \mathrm{CH}_{3}$ & $4.02, \mathrm{dd}(13.4,3.9) ; 4.83, \mathrm{dd}(13.4,4.5)$ & $62.4, \mathrm{CH}_{2}$ & $4.48, \mathrm{dd}(12.7,3.3) ; 4.32, \mathrm{~m}$ & $61.7, \mathrm{CH}_{2}$ \\
\hline 28 & $1.47, \mathrm{~s}$ & $26.4, \mathrm{CH}_{3}$ & $1.30, \mathrm{~s}$ & $26.1, \mathrm{CH}_{3}$ & $1.45, \mathrm{~s}$ & $31.7, \mathrm{CH}_{3}$ \\
\hline $\mathrm{OMe}$ & & & & & $3.27, \mathrm{~s}$ & $55.7, \mathrm{CH}_{3}$ \\
\hline
\end{tabular}

${ }_{\text {in pyridine- }} d_{5}$, bin $\mathrm{CDCl}_{3}$.

bridge was ruptured. This was further determined by the HMBC correlations (Figure 1$)$ from Me-27 $\left(\delta_{\mathrm{H}} 1.29\right)$ to C-25 $\left(\delta_{\mathrm{C}}\right.$ 42.4) and $\mathrm{C}-26\left(\delta_{\mathrm{C}}\right.$ 173.0). Moreover, the HMBC correlations from $\delta_{\mathrm{H}} 1.65(\mathrm{H}-19), 6.24(\mathrm{H}-2)$, and $7.19(\mathrm{H}-3)$ to $\delta_{\mathrm{C}} 202.9(\mathrm{C}-1)$ demonstrated the presence of an conjugated 2-en-1-one group, and the ${ }^{1} \mathrm{H}-{ }^{1} \mathrm{H}$ COSY correlations of $\delta_{\mathrm{H}} 7.19$ $(\mathrm{H}-3) / \delta_{\mathrm{H}} 4.01(\mathrm{H}-4)$ revealed that there existed an hydroxyl group at C-4. An oxymethine in $\mathbf{1}$ instead of a methene in physalin $\mathrm{J}$ suggests that one hydroxyl group could be located at $\mathrm{C}-7$, which was confirmed by the ${ }^{1} \mathrm{H}-{ }^{1} \mathrm{H}$ COSY correlations of $\delta_{\mathrm{H}} 3.54(\mathrm{H}-6) / \delta_{\mathrm{H}} 5.70(\mathrm{H}-7)$ and $\delta_{\mathrm{H}} 5.70(\mathrm{H}-7) / \delta_{\mathrm{H}} 2.93(\mathrm{H}-8)$. The relative configurations of $\mathrm{H}-8$ and $\mathrm{Me}-28$ in the physalin skeleton were established to be $\beta$-oriented by X-ray. ${ }^{20,21}$ Thus, the NOE correlations (Figure 1) of H-4/H-6, H-6/H-7, H-7/H$8 \beta$, and $\mathrm{H}-27 / \mathrm{Me}-28$ established the relative configuration of $\mathrm{OH}-4$ and $\mathrm{OH}-7$ to be $\alpha$-oriented and Me- 27 to be $\beta$-oriented. Hence, the structure of compound 1 was identified to be physalin III (1).
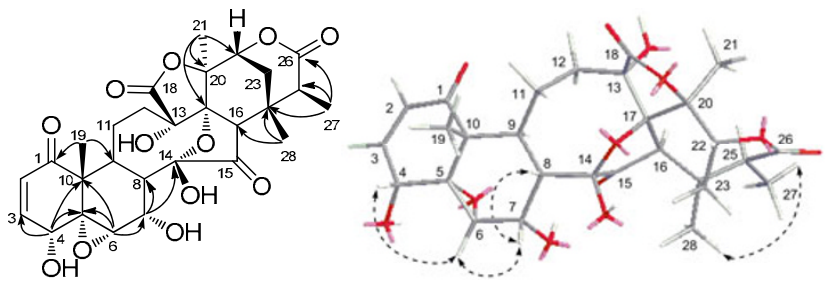

Figure 1. Key HMBC $(\mathrm{H} \rightarrow \mathrm{C})$ and $\operatorname{ROESY}(\mathrm{H} \leftrightarrow \mathrm{H})$ correlations of compound 1

Compound 2 was obtained as a colorless needle crystal. The molecular formula of 2 was determined to be $\mathrm{C}_{28} \mathrm{H}_{32} \mathrm{O}_{12}$ on the basis of HREIMS ( $\mathrm{m} / \mathrm{z} 560.1854,[\mathrm{M}]^{+}$; calcd 560.1894) and NMR data (Table 1). The NMR data of 2 were similar to those of physalin $Z^{22}$, and the differences between them were two of the double-bond carbon signals in physalin $\mathrm{Z}$ were replaced by two oxygenated methine signals in 2 located at C- 6 and C-7, which were deduced by ${ }^{1} \mathrm{H}-{ }^{1} \mathrm{H}$ COSY correlations of $\mathrm{H}-8\left(\delta_{\mathrm{H}}\right.$ $3.39) / \mathrm{H}-7\left(\delta_{\mathrm{H}} 5.23\right)$ and $\mathrm{H}-7\left(\delta_{\mathrm{H}} 5.23\right) / \mathrm{H}-6\left(\delta_{\mathrm{H}} 4.79\right)$ and by HMBC correlations from $\mathrm{H}-6$ to C-10, C-4, C-8, C-7, and C-5, from $\mathrm{H}-7$ to $\mathrm{C}-6, \mathrm{C}-5, \mathrm{C}-8$, and $\mathrm{C}-14$. The ROESY correlations (Figure 2) of $\mathrm{H}-6 / \mathrm{H}-7$ and $\mathrm{H}-7 / \mathrm{H}-8 \beta$ revealed that $\mathrm{OH}-\mathrm{C}(6)$ and $\mathrm{OH}-\mathrm{C}(7)$ were $\alpha$-oriented. So the structure of compound 2 was deduced and named as physalin IV (2).

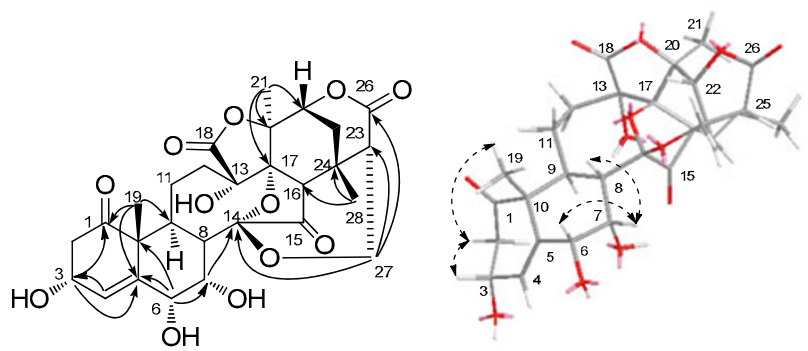

Figure 2. Key HMBC $(\mathrm{H} \rightarrow \mathrm{C})$ and $\operatorname{ROESY}(\mathrm{H} \leftrightarrow \mathrm{H})$ correlations of compound 2

Compound 3 was obtained as an amorphous yellow powder. The molecular formula of $\mathbf{3}$ was determined as $\mathrm{C}_{29} \mathrm{H}_{32} \mathrm{O}_{10}$ using HREIMS and NMR data (Table 1). These NMR data of 3 were similar to those of physalin $X^{23}$, except the presence of an additional methoxyl group. Comparing with physalin $\mathrm{X}$, the chemical shift of C-3 $\left(\delta_{\mathrm{C}} 76.7\right)$ at down field and the HMBC correlation (Figure 3 ) of OMe $\left(\delta_{\mathrm{H}} 3.27\right)$ with $\mathrm{C}-3$ in 3 indicated a methoxyl group at $\mathrm{C}-3$. The relative configuration of Me-19 and H-16 in physalin skeleton were established to be $\beta$ oriented by X-ray. ${ }^{20,21}$ So the relative configuration of $3-\mathrm{OMe}$ could be determined as $\alpha$-oriented by the NOE correlations of Me-19/H-3 and H-16/H-3. Hence, the structure of compound 3 was identified to be 3-O-methylphysalin X (3). 
The structures of five known steroidal compounds, physalin $\mathrm{Z}(\mathbf{4})^{22}$, physalin $\mathrm{C}(\mathbf{5})^{24}$, physalin I $(\mathbf{6})^{25}$, physalin $\mathrm{P}(\mathbf{7})^{26}$, 25,27-dihydro-4,7-dedehydro-7-deoxyneophysalin A $(\mathbf{8})^{27}$, were elucidated in comparison to their spectroscopic data with those reported in the literature.

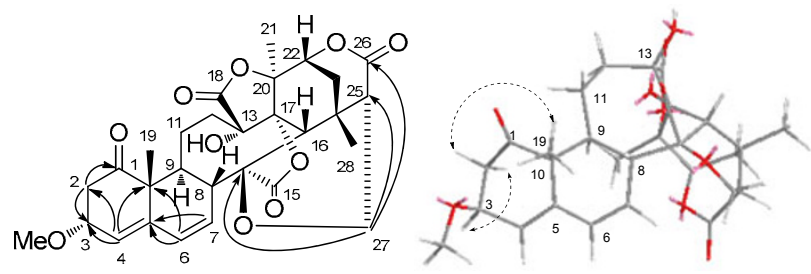

Figure 3. Key HMBC $(\mathrm{H} \rightarrow \mathrm{C})$ and $\operatorname{ROESY}(\mathrm{H} \leftrightarrow \mathrm{H})$ correlations of compound $\mathbf{3}$

\section{Experimental Section}

General Experimental Procedures. Column chromatography (CC): silica gel (100-200 mesh, 200-300 mesh; Qingdao Marine Chemical Co., Ltd.), Sephadex LH-20 (Pharmacia). Thin-layer chromatography (TLC): silica gel $60 \mathrm{~F}_{254}$ (Qingdao Marine Chemical Co., Ltd.). Optical rotations: Jasco P-1020 polarimeter. UV Spectra: Shimadzu UV-2401 PC spectraphotometer. IR Spectra: Bruker Tensor-27 infrared spectrophotometer, with $\mathrm{KBr}$ pellets in $\mathrm{cm}^{-1}$. NMR Spectra: Bruker Bruker Avance III-600MHz spectrometer; chemical shifts $\delta$ were recorded as ppm relative to TMS, coupling constants $J$ in Hz. ESIMS: API QSTAR Pulsar spectrometer; HREIMS: Waters Autospec Premier P776.

Plant Material. The calyces of Phsalis alkekengi var. franchetii were purchased in Kunming CTM market, Kunming city, Yunnan province at December 2011 and were identified by Prof. Xi-Wen Li who was botanic taxonomist in Kunming Institute of Botany.

Extraction and Isolation. Dried calyces $(15 \mathrm{~kg})$ of $P$. alkekengi var. franchetii were extracted with $80 \%$ EtOH under reflux. The extract was suspended in water $(5 \mathrm{~L})$ and then partitioned successively with $\mathrm{PE}$ (petroleum ether) $(8 \mathrm{~L} \times 4)$ and EtOAc $(8 \mathrm{~L} \times 5)$. The EtOAc-soluble layer (evaporated under vacuum to get $250 \mathrm{~g}$ ) eluted successively with $\mathrm{CHCl}_{3}$, $\mathrm{CHCl}_{3}-\mathrm{MeOH}(100: 1), \mathrm{CHCl}_{3}-\mathrm{MeOH}(20: 1)$, and $\mathrm{CHCl}_{3}-$ $\mathrm{MeOH}(5: 1)$. Then, the $\mathrm{CHCl}_{3}-\mathrm{MeOH}(100: 1)$ portion (80 g) was chromatographed on a silica gel column using a gradient of $\mathrm{CHCl}_{3}-\mathrm{CH}_{3} \mathrm{OH}$ (100:0 to 0:100), which yielded five fractions (1-5). Fraction 2 was successively subjected to RP-18, Sephadex LH-20 and silica gel to derive subfractions I-IV. Subfraction II was further separated over silica gel with $\mathrm{CHCl}_{3}-\mathrm{CH}_{3} \mathrm{OH}(120: 1)$ to yield compound $3(5 \mathrm{mg}), \mathbf{6}(4 \mathrm{mg})$, $7(10 \mathrm{mg})$ and $8(3 \mathrm{mg})$. Subfraction III was subjected to semipreparative HPLC $\left(\mathrm{CH}_{3} \mathrm{CN}-\mathrm{H}_{2} \mathrm{O}, 50: 50\right)$ to produce $4(1 \mathrm{mg})$ and 5 (1 mg). Fraction 3 was successively subjected to RP-18, Sephadex LH-20 and separated over silica gel with $\mathrm{CHCl}_{3}$ $\mathrm{CH}_{3} \mathrm{OH}(80: 1)$ to yield $\mathbf{1}(2 \mathrm{mg})$ and $2(6 \mathrm{mg})$.

Physalin III (1): white powder; $[\alpha]_{\mathrm{D}}^{21}-29.33$ (c 0.2 , $\mathrm{CHCl}_{3}$ ); UV $\left(\mathrm{CHCl}_{3}\right): \lambda_{\max }(\log \varepsilon): 240(3.36) \mathrm{nm}$; IR (KBr): $v_{\max } 3430,2924,1787,1758,1728,1462,1376,1218,1073$ $1041 \mathrm{~cm}^{-1}$; ${ }^{1} \mathrm{H}$ and ${ }^{13} \mathrm{C}$ NMR: see Table 1 . Negative ESIMS: $559\left([\mathrm{M}-\mathrm{H}]^{-}\right)$. HREIMS: $560.1885[\mathrm{M}]^{+}$(calcd. for $\left.\mathrm{C}_{28} \mathrm{H}_{32} \mathrm{O}_{12}, 560.1894\right)$.

Physalin IV (2): colorless needle crystal $\left(\mathrm{CHCl}_{3}\right) ;[\alpha]_{\mathrm{D}}^{16}-$ 114.40 (c 0.1, MeOH); UV (MeOH): $\lambda_{\max }(\log \varepsilon): 201$ (4.23) $\mathrm{nm}$; IR (KBr): $v_{\max } 3431,1781,1729,1632,1383,1374,1167$, 1134, $1065 \mathrm{~cm}^{-1} ;{ }^{1} \mathrm{H}$ and ${ }^{13} \mathrm{C}$ NMR: see Table 1. Positive ESIMS: $583\left([\mathrm{M}+\mathrm{Na}]^{+}\right)$. HREIMS: $560.1854[\mathrm{M}]^{+}$(calcd. for $\left.\mathrm{C}_{28} \mathrm{H}_{32} \mathrm{O}_{12}, 560.1894\right)$.

3-O-Methylphysalin X (3): yellow powder; $[\alpha]_{\mathrm{D}}^{18}-16.00$ (c 0.1, pyridine); UV $(\mathrm{MeOH}): \lambda_{\max }(\log \varepsilon): 225$ (3.77), 201 (3.80) $\mathrm{nm}$; IR (KBr): $v_{\max } 3432,2923,1786,1629 \mathrm{~cm}^{-1} ;{ }^{1} \mathrm{H}$ and ${ }^{13} \mathrm{C}$ NMR: see Table 1. Positive ESIMS: $563\left([\mathrm{M}+\mathrm{Na}]^{+}\right)$. HREIMS: $540.1933[\mathrm{M}]^{+}$(calcd. for $\mathrm{C}_{29} \mathrm{H}_{32} \mathrm{O}_{10}, 540.1995$ ).

\section{Electronic Supplementary Material}

Supplementary material is available in the online version of this article at http://dx.doi.org/10.1007/s13659-013-0021-z and is accessible for authorized users.

\section{Acknowledgments}

This project was financially supported by the National Major Basic Research Program (No. SB2007FY400) of the National Ministry of Science and Technology of China, the National Knowledge Innovation Program of Chinese Academy of Sciences (No. KSCX2-YW-G-038), and the Foundation of State Key Laboratory of Phytochemistry and Plant Resources in West China (P2010-ZZ14).

Open Access This article is distributed under the terms of the Creative Commons Attribution License which permits any use, distribution, and reproduction in any medium, provided the original author(s) and source are credited.

\section{References}

[1] Kuang, K. R. Flora Republicae Popularis Sinicae (Zhongguo Zhiwu Zhi) 1978, 67, 10.

[2] Committee, N. P. Pharmacopeia of People's Republic of China (the 2010 edition) 2010, 1, 337-338.

[3] Wu, H. Y.; Suo, Q. L.; Li, J. Neimenggu Shiyou Huagong 2007, 4, $5-8$.

[4] Zhang, N.; Bie, Z. M.; Qing, W. J.; Zhang, Y.; Ge, H. J.; Shu, C.; Shi, H. G.; Ma, H. B. Journal of Jilin Medical College 2008, 29, 104-107.

[5] Kawai, M.; Matsuura, T.; Taga, T.; Osaki, K. J. Chem. Soc. B 1970, 812-815.

[6] Kawai, M.; Makino, B.; Taga, T.; Miwa, Y.; Yamamoto, T.; Furuta, T.; Yamamura, H.; Butsugan, Y.; Ogawa, K.; Hayashi, M. Bull. Chem. Soc. Jpn. 1994, 67, 222-226.

[7] Taga, T.; Miwa, Y.; Machida, K.; Kawai, M.; Buisugan, Y. Acta Crystallogr. C 1991, 47, 2188-2191.

[8] Kawai, M.; Ogura, T.; Butsugan, Y.; Taga, T.; Hayashi, M. Tetrahedron 1991, 47, 2103-2110.

[9] Han, H.; Qiu, L.; Wang, X.; Qiu, F.; Wong, Y.; Yao, X. Biol. Pharm. Bull. 2011, 34, 1584-1588.

[10] Silva, M. T. G.; Simas, S. M.; Batista, T. G. F. M.; Cardarelli, P.; Tomassini, T. C. B. Mem. Inst. Oswaldo Cruz 2005, 100, 
779-782.

[11] Sa, M. S.; de Menezes, M. N.; Krettli, A. U.; Ribeiro, I. M.; Tomassini, T. C. B.; Ribeiro, D. S. R.; de Azevedo, W. F.; Soares, M. B. P. J. Nat. Prod. 2011, 74, 2269-2272.

[12] Yu, Y.; Sun, L.; Ma, L.; Li, J.; Hu, L.; Liu, J. Int. Immunopharmacol. 2010, 10, 290-297.

[13] Pinto, N. B.; Morais, T. C.; Carvalho, K. M. B.; Silva, C. R.; Andrade, G. M.; Brito, G. A. C.; Veras, M. L.; Pessoa, O. D. L.; Rao, V. S.; Santos, F. A. Phytomedicine 2010, 17, 740-743.

[14] Budhiraja, R. D.; Sudhir, S.; Garg, K. N. Planta Med. 1984, 50, 134-136.

[15] Lin, Y. S.; Chiang, H. C.; Kan, W. S.; Hone, E.; Shih, S. J.; Won, M. H. Am. J. Chin. Med. 1992, 20, 233-243.

[16] Habtemariam, S. Planta Med. 1997, 63, 15-17.

[17] Abe, F.; Nagafuji, S.; Okawa, M.; Kinjo, J. Chem. Pharm. Bull. 2006, 54, 1226-1228.

[18] Vieira, N. C.; Espindola, L. S.; Santana, J. M.; Veras, M. L.; Pessoa, O. D. L.; Pinheiro, S. M.; Mendonca, d. A. R.; Lima, M. A. S.; Silveira, E. R. Bioorg. Med. Chem. 2008, 16, 1676-1682.

[19] Row, L. R.; Sarma, N. S.; Reddy, K. S.; Matsuura, T.; Nakashima, R. Phytochemistry 1978, 17, 1647-1650.
[20] Kawai, M.; Matsuura, T.; Taga, T.; Osaki, K. J. Chem. Soc. B 1970, 812-815.

[21] Kawai, M.; Makino, B.; Taga, T.; Miwa, Y.; Yamamoto, T.; Furuta, T.; Yamamura, H.; Butsugan, Y.; Ogawa, K.; Hayashi, M. Bull. Chem. Soc. Jpn. 1994, 67, 222-226.

[22] Qiu, L.; Zhao, F.; Jiang, Z. H.; Chen, L. X.; Zhao, Q.; Liu, H. X.; Yao, X. S.; Qiu, F. J. Nat. Prod. 2008, 71, 642-646.

[23] Chen, R.; Liang, J. Y.; Liu, R. Helv. Chim. Acta. 2007, 90, $963-$ 966.

[24] Kawai, M.; Matsuura, T. Tetrahedron 1970, 26, 1743-1745.

[25] Row, L. R.; Reddy, K. S.; Sarma, N. S.; Matsuura, T.; Nakashima, R. Phytochemistry 1980, 19, 1175-1181.

[26] Kawai, M.; Matsumoto, A.; Makino, B.; Mori, H.; Ogura, T.; Butsugan, Y.; Ogawa, K.; Hayashi, M. Bull. Chem. Soc. Jpn. 1993, 66, 1299-1300.

[27] Sunayama, R.; Kuroyanagi, M.; Umehara, K.; Ueno, A. Phytochemistry 1993, 34, 529-533. 